\title{
Effect of a topical treatment in organotypic culture of human breast skin after exposure to gamma-rays
}

\author{
N. Gagliano, ${ }^{1}$ M. Bedoni, ${ }^{2}$ G. Mantovani, ${ }^{2}$ M. Chiriva-Internati, ${ }^{3}$ D. Castelli, ${ }^{2}$ C. Torri, $^{1}$ E. Donetti ${ }^{2}$ \\ ${ }^{1}$ Department of Human Morphology, Extracellular Matrix Laboratory-EML, University of Milan, Milan, Italy; \\ ${ }^{2}$ Department of Human Morphology, Laboratory of Immunohistochemistry of Epithelia, University of \\ Milan, Milan, Italy; ${ }^{3}$ Department of Microbiology \& Immunology, Divisions of Hematology \& Oncology, \\ Texas Tech University Health Sciences Center and Southwest Cancer Treatment and Research Center, \\ Lubbock, TX, USA
}

(C)2007 European Journal of Histochemistry

The early radiation of epidermal reactions can lead to healing of the lesion or radiation necrosis. There is no general agreement for either the prevention and/or treatment of radiation skin response, also as little is known about the immediate phases of this phenomenon. We investigated the early effects exerted by Healing and Wound Emulsion (HWE) on human skin response after ionizing radiation. Epidermal morphology, Heat Shock Protein (HSP) 70, and Transforming Growth Factor- $\beta 1$ (TGF- $\beta 1$ ) gene expression were investigated in organotypic human skin cultures undergoing a double dose of gamma-rays (2 Gy). HSP70 gene expression tended to be induced in the HWE group 6 hours after cream administration and was significantly up-regulated after 48 hours, when epidermal morphological alterations were evident. TGF$\beta 1$ seems not affected in cream treated samples. HWE may stimulate skin to mount an early defensive response against damage induced by gamma rays.

Key words: radiodermatitis, epidermis, ionizing radiations, TGF- $\beta 1$, HSP70.

Correspondence: Nicoletta Gagliano,

Department of Human Morphology, Extracellular Matrix Laboratory-EML, University of Milan, Via Fratelli Cervi, 93 20090 Segrate, Milan, Italy

Tel: +39.02.50330462.

Fax: +39.02 .50330452$

E-mail: nicoletta.gagliano@unimi.it

Paper accepted on November 16, 2007

European Journal of Histochemistry

2007; vol. 51 issue 4 (October-December):283-290
$\mathrm{R}$ adiotherapy is employed in approximately 50 to 60 percent of patients with cancer both to control the tumour and for the management of tumour-related symptoms (Porock et al., 2002).

Radiotherapy induces reactions in many of the treated patients; one of the most common side effects is an acute skin response, referred to as radiodermatitis, which affects up to $95 \%$ of patients (De Conno et al., 1991; King et al., 1985). Acute radiation reactions typically appear 10-14 days after radiotherapy, continue to increase in severity until the completion of treatment (Porock et al., 1999), and may persist for up to 4 weeks after the end of treatment. These early effects are characterized by epilation and erythema, followed by dry and moist desquamation of the epidermis that can lead to either healing of the lesion or radiation necrosis (Porock et al., 1999; Sivan et al., 2002). These side effects induce a condition ranging from mild irritation to severe pain, and may require a temporary or permanent withdrawal of the therapy (Williams et al., 1996). During the next few months or years, late effects appear, characterized by fibrosis which involves the connective compartment of the skin.

In order to minimize the effects of acute skin reactions, a number of management strategies have been suggested. At present, there is no general agreement for the prevention and/or treatment of radiation skin response (Barkham 1993; Lavery 1995; Schmuth et al., 2002; for review see Bolderston et al., 2006); most managements rely on topical skin treatments. However, these treatments vary among cancer institutions and are not based upon principles but rather upon historical practice and personal preference. Currently, management is focused on the attempt to prevent the skin deterioration to higher grades of dermatitis, to relieve the pain and itch, and on addressing any infections. This is done by advising 
patients to keep the skin clean and moist throughout their radiotherapy treatment and afterwards.

The assessment of the early skin response to gamma-rays is a difficult endeavour mainly for ethical reasons, and, therefore, experimental models mimicking the clinical setting are needed in defining this issue. Recently, we have characterized in our laboratory an experimental model of human organotypic skin cultures, which offers the advantage of the tridimensional arrangement and of epithelialmesenchimal interactions (Bedoni et al., 2007).

Using this model, we found a marked inhibition of epidermal proliferation after a single standard clinical dose of gamma-rays (2 Gy) within the first 24 hours (Donetti et al., 2005), indicating that skin homeostasis is rapidly altered by ionizing rays.

The aim of this study was to investigate the main morphological features of the epithelial compartment and to perform molecular analysis of specific target genes in organotypic human skin cultures undergoing a double standard clinical dose of gamma-rays (2 Gy) after the application of an innovative compound, Healing and Wound Emulsion (HWE, provided by Sinclair Pharma Group). As target genes, we analyzed the expression of transforming growth factor beta 1 (TGF- $\beta 1$ ) and of the heat shock protein (HSP) 70. TGF- $\beta 1$ is a multifunctional cytokine involved in the maturation of epidermis, which promotes the initiation and differentiation of keratinocytes by blocking their proliferation (Barkham 1993). HSP70 is expressed in the skin and induced under stressful conditions (Daugaard et al., 2007), thus representing a useful stress marker after ionizing radiation.

The main components of the cream HWE are hyaluronic acid, vitis vinifera, shea butter, telmesteine, glycyrrhetinic acid, and bisabolol in a hydrolipidic base.

\section{Materials and Methods}

\section{Organotypic skin cultures}

Human breast skin samples were obtained from cosmetic surgery of 30-40 year old healthy women $(n=4)$ after informed consent, accordingly with the Helsinki declaration. Immediately after excision, skin was transported in cold culture medium and cut into $0.5-1 \mathrm{~cm}^{2}$ fragments using a scalpel. Skin biopsies were cultured in a Transwell system (Costar, Corning Inc, NY, USA) at $37^{\circ} \mathrm{C}$ and $5 \%$ $\mathrm{CO}_{2}$ overnight to reduce the acute effects of explan- tation, as previously described (Donetti et al., 2005; Bedoni et al., 2007). All skin fragments were cultured in DMEM culture medium containing $10 \%$ foetal bovine serum (FBS) (Invitrogen, Milan, Italy) supplemented with hydrocortisone, amphotericine B, and glutamine (Sigma Aldrich, Milan, Italy). Skin biopsies were placed epidermal side up at the air liquid interface. Medium was changed every day and the stratum corneum remained constantly exposed to the air. After the overnight incubation, samples were divided in irradiated and non irradiated groups. Each donor was represented in triplicate in all experimental groups at all considered time points.

\section{Gamma-irradiation protocol}

All irradiated samples were twice exposed to a 2 Gy dose emitted by ${ }^{137} \mathrm{Cs}$-irradiator, with the second irradiation administered 24 hours after the first one, when irradiated samples were further divided in two experimental groups: IRR and HWE groups. The IRR group was only irradiated, whilst HWE group was irradiated and then HWE cream was applied on the epidermal surface for one hour after each irradiation. At the end of the HWE application, samples were thoroughly washed with phosphate buffer saline (PBS) $0.1 \mathrm{M}, \mathrm{pH} 7.4$ and were maintained in Transwell systems either for 6 or 48 hours after the second irradiation.

Non irradiated skin biopsies (CT) were kept at room temperature during gamma-irradiation and incubated for 6 or 48 hours after the second irradiation under the same conditions, representing controls.

$C T, I R R$, and HWE skin fragments harvested at the different time points were processed either for the morphological analysis or immediately frozen in liquid nitrogen and stored at $-80^{\circ} \mathrm{C}$ until use.

\section{Morphological analysis}

For each donor, 2 replicates/treatments were fixed in $4 \%$ formalin in $0.1 \mathrm{M} \mathrm{PBS}(\mathrm{pH} \mathrm{7.4)}$ for 5 hours at room temperature, routinely dehydrated, paraffin embedded, and $5 \mu \mathrm{m}$ serial sections were obtained. Haematoxilyn-eosin stained sections were analyzed by two independent investigators, using a light microscopy Nikon Eclipse E600 equipped with a Nikon digital camera DXM1200 (Nikon, Tokyo, Japan).

\section{PCR analysis}

For each donor, 1 frozen replicate/treatment was 
homogenized with an Ultraturrax and total RNA was extracted by a modification of the guanidineisothiocyanate method (Tri-Reagent, Sigma, Milan, Italy). Total RNA was obtained for each single skin fragment obtained from each donor. Total RNA ( 1 $\mu \mathrm{g}$ ) was DNase digested, and first-strand DNA was carried out following the manufacturer's protocol (Promega Italia, Milan, Italy). PCR amplification was performed with primers specific for TGF- $\beta 1$ and HSP70.28s was used as an internal control.

Primer sequences were the following: TGF- $\beta 1$ forward CAG AAA TAC AGC AAC AAT TCC TGG, reverse TTG CAG TGT GTT ATC CCT GCT GTC (186 bp); HSP70 forward CGACCTGAACAAGAGCATCA, reverse AAGATCTGCGTCTGCTTGGT (213 bp); 28s forward TTA CCA AAA GTG GCC CAC TA, reverse GAA AGA TGG TGA ACT ATG CC (345 bp). PCR products were resolved by electrophoresis in $2 \%$ agarose gels, stained with ethidium bromide and quantified by densitometric analysis. TGF- $\beta 1$ and HSP70 mRNA levels were normalized on $28 \mathrm{~s}$ gene expression.

\section{Statistical analysis}

Experimental groups were compared by one-way ANOVA, followed by the Student-Neumann-Keuls post test. Results are expressed as mean \pm SEM. A $p$ value $<0.05$ was considered significant.

\section{Results}

\section{Morphological analysis}

A quantitative assessment of morphological changes between IRR and HWE groups at different time points is reported in Table 1. Among the different experimental groups, no evident differences were detected in the overall distribution of the basal brownish pigmentation due to melanin.

6 hours. Light microscopic analysis of the skin samples of the IRR group harvested 6 hours after the double gamma-irradiation revealed that epidermal architecture was not yet affected (Figure 1a) compared to the CT group (Figure $1 b$ ). In both experimental groups, a profound basal monolayer composed of regular cylindrical keratinocytes, multilayered spinous/granular layer, and an outer stratum corneum were well preserved. Compared to the IRR group, the HWE samples showed a slight enlargement of intercellular spaces (arrowheads in Figure 1c). Scattered keratinocytes with high condensed chromatin and pale cytoplasm were present
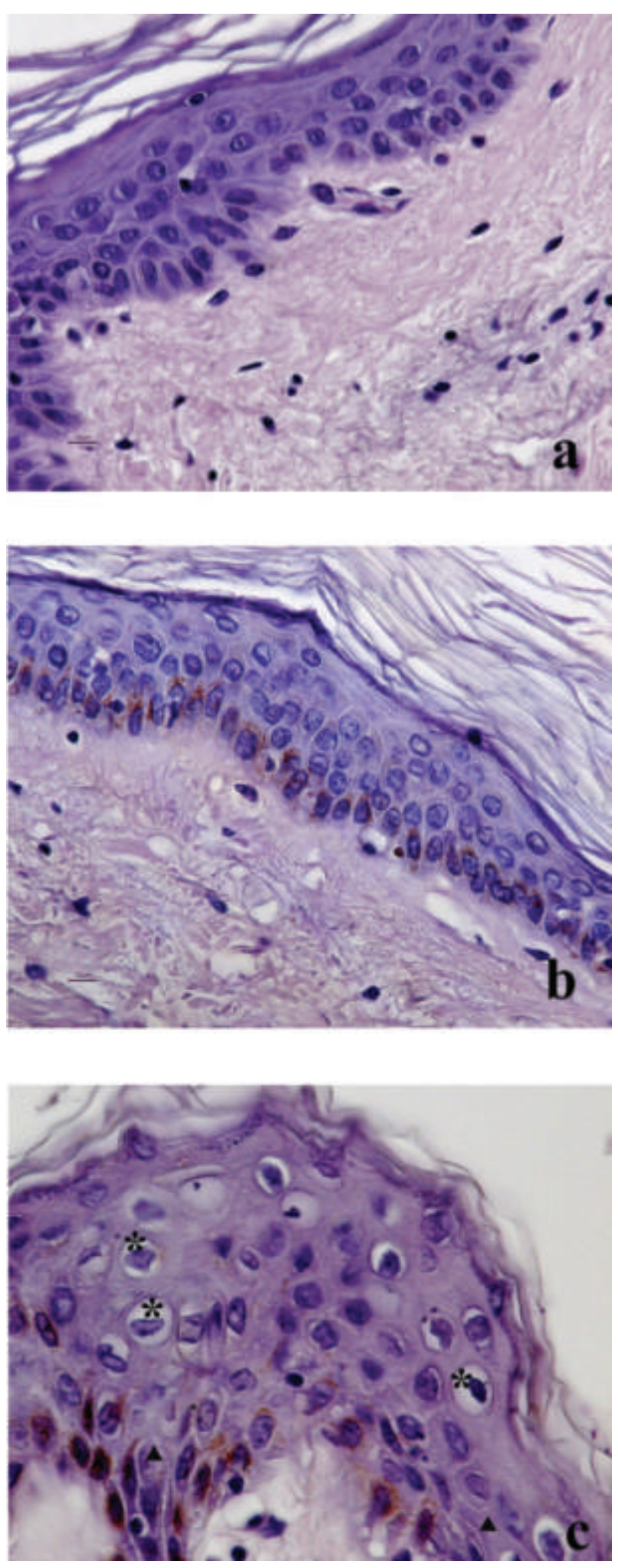

Figure 1. Photomicrographs of haematoxylin/eosin stained paraffin sections ( $5 \mu \mathrm{m}$ thick) of human breast skin harvested 6 hours after a double dose of $\mathbf{2}$ Gy of gamma-rays. (a) Irradiated epidermis; (b) non irradiated epidermis; (c) irradiated epidermis with HWE. In (c), asterisks indicate keratinocytes with nuclear condensation and pyknosis, and arrowheads show enlargement of intercellular spaces. Original magnification 40X, bar $10 \mu \mathrm{m}$. 
in the basal/suprabasal epidermal layers (see asterisks in Figure 1c).

48 hours. When comparing the epithelial morphological features of the IRR and CT groups, a slight increase of basal/suprabasal pyknosis were observed in the former samples (compare Figure $2 \mathrm{a}$ and $2 \mathrm{~b}$ ). In all irradiated samples harvested 48 hours after the double gamma-irradiation, a moderate widening of intercellular spaces occurred throughout all the epithelium. At this time point, in HWE group, epidermis was present, but nuclear pyknosis massively occurred in the suprabasal layers (Figure 2c).

\section{Molecular biology analysis}

TGF- $\beta 1$ gene expression: the effect of HWE on TGF- $\beta 1$ gene expression after gamma-radiations is shown in Figure 3a.TGF- $\beta 1$ mRNA levels, although with some interindividual differences, were similarly expressed in all the experimental groups at the time points investigated.

HSP70 gene expression: 6 hours after irradiation, the administration of HWE appeared to induce HSP70 gene expression (Figure 3b), while 48 hours after radiations, the treatment with HWE induced a significant level of HSP70 mRNA levels $(p<0.05$ for HWE vs CT and IRR) (Figure $3 c$ ).

\section{Discussion}

The knowledge of the early acute epidermal responses after ionizing radiation induced by radiotherapy might elucidate the mechanisms leading to late damage (Archambeau et al., 1995) and may contribute to the prevention and/or treatment of the radiation skin response. The early response induced by environmental damaging stimuli can be

Table 1. Morphological evaluation of epidermal features in irradiated samples.

\begin{tabular}{lccc}
\hline Time & Parameter & IRR group & HWE group \\
\hline \multirow{4}{*}{6 hours } & Basal pyknosis & \pm & ++ \\
& Suprabasal pyknosis & \pm & ++ \\
& Widening of intercellular spaces & \pm & ++ \\
48 hours & Basal pyknosis & \pm & ++ \\
& Suprabasal pyknosis & \pm & ++ \\
& Widening of intercellular spaces & ++ & +++ \\
\hline
\end{tabular}

IRR group: irradiated skin; HWE group: irradiated and HWE treated skin after each irradiation (see Methods section for details). Histological assessment was scored as follows: 士: scattered; ++: moderate; +++: severe.
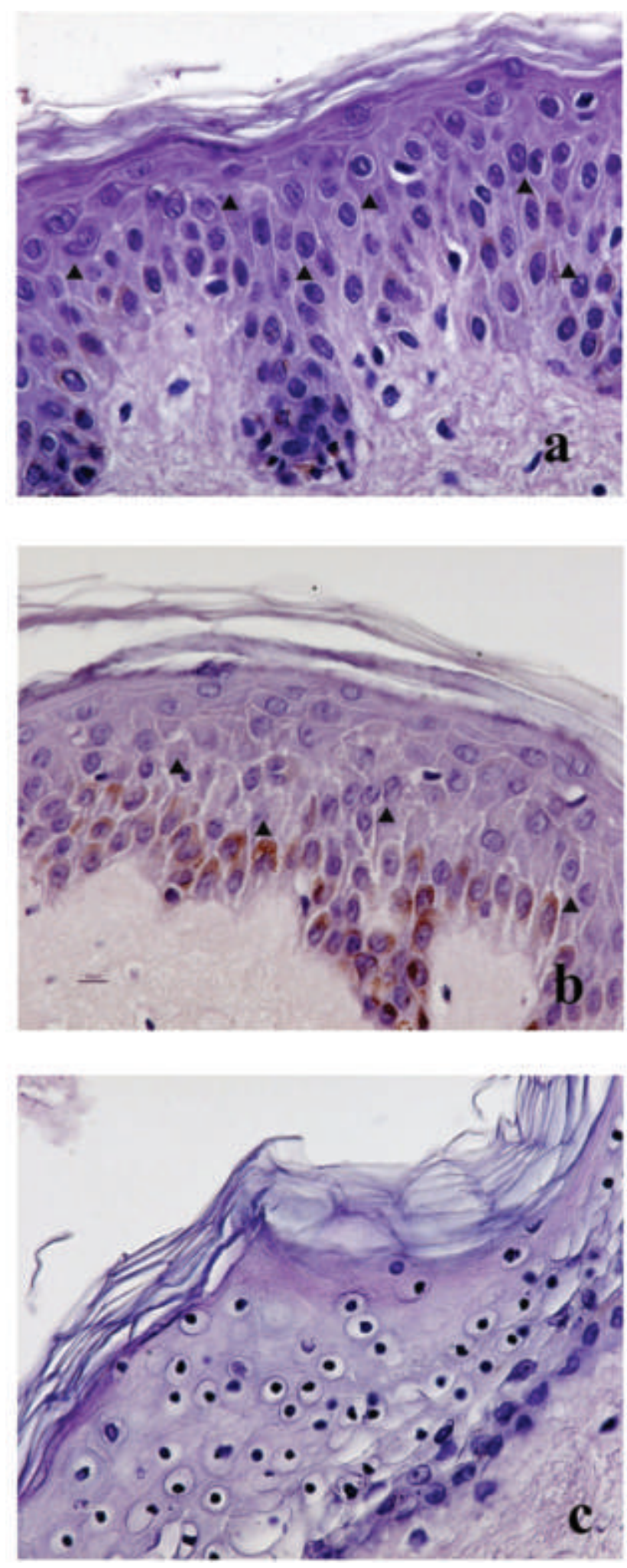

Figure 2. Photomicrographs of haematoxylin/eosin stained paraffin sections ( $5 \mathrm{Im}$ thick) of human breast skin harvested 48 hours after a double dose of 2 Gy of gamma-rays. (a) Irradiated epidermis; (b) non irradiated epidermis; (c) irradiated epidermis with HWE. In (a) and (b) arrowheads show enlargement of intercellular spaces. Original magnification 40x, bar $10 \mu \mathrm{m}$. 
a)

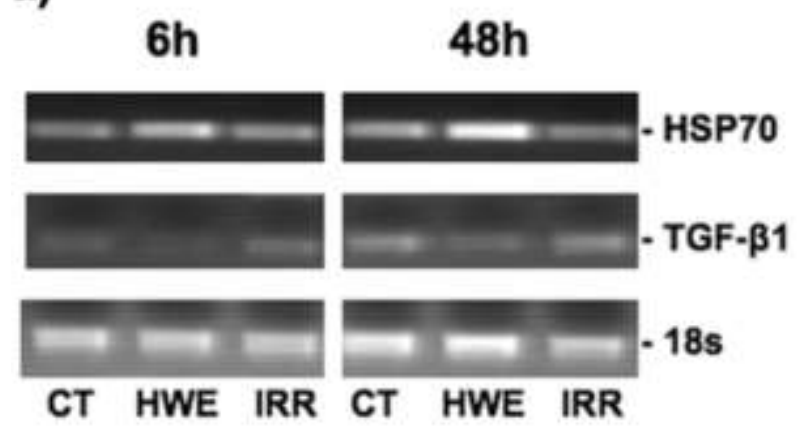

b)

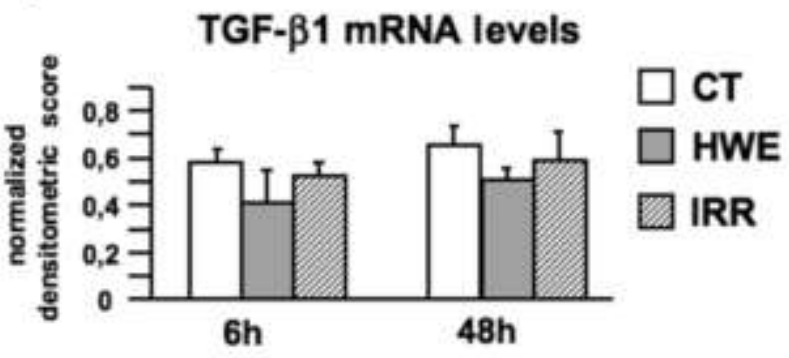

c)

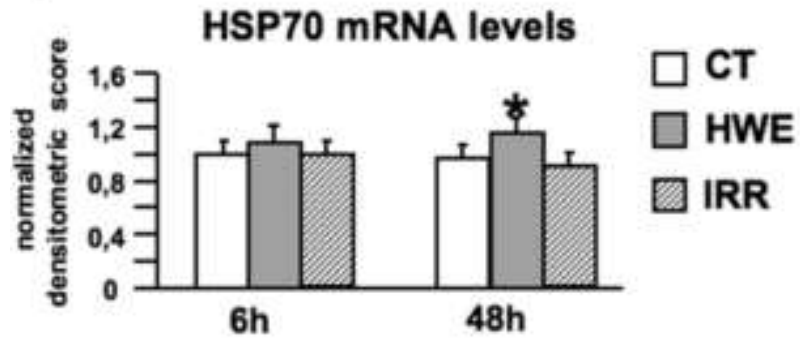

Figure 3. a) Representative RT-PCR for TGF-,1 and HSP70. Bar graphs showing TGF- $\beta 1$ (b) and HSP70 gene expression (c) in human breast skin harvested 6 and 48 hours after a double dose of 2 Gy of gamma-rays. TGF- $\beta 1$ and HSP70 mRNA levels are expressed as normalized densitometric units in relation to $28 \mathrm{~s}$ gene expression. Data are expressed as normalized densitometric score. ${ }^{\star} p<0.005$ vs CT, HWE and IRR groups.

investigated in skin organotypic cultures which have been already characterized (Bedoni et al., 2007) and could be exploited as a good tool to investigate the whole dermal/epidermal events (Moll et al., 1999; Bäckvall et al., 2002; Donetti et al., 2005). The maintenance of the three dimensional arrangement is pivotal since the cross-talking between the epidermal and dermal compartments of the human skin plays a key role in physiological and pathological conditions.
To our knowledge, no experimental evidence is available about i) the morphological epithelial features and molecular mechanism(s) induced in human skin and ii) the effect of a topical treatment immediately after exposure to ionizing rays. The present study represents the first effort to elucidate these issues.

The evaluation of HSP70 gene expression gave insights into the effect of HWE in the early phases of skin stress response. Ionizing radiation induces a skin stress response, similarly to elevated temperatures, alcohol, heavy metals and oxidants. All organisms respond to such stimuli with the transcription of Heat Shock Proteins (HSPs), among which HSP70 is the most abundant and investigated member (Lindquist 1986, Maytin 1995). The transient induction of $\mathrm{HSPs}$, in fact, produces a state of increased resistance to further stress and the heat shock response is generally thought to represent an adaptive mechanism to counteract adverse environmental conditions. Like in any other organ system, HSPs and, in particular HSP70, are expressed in the skin: under stressful conditions they are induced in epidermal and dermal cells (Lindquist 1986; Kwon et al., 2002).

As early as 6 hours after the double exposure to gamma-rays, cream application slightly increased HSP70 mRNA levels. This observation suggests that $\mathrm{HWE}$ rapidly stimulates the skin to produce a response for counteracting a noxious stimulus, as gamma rays. Interestingly, at longer time points (48h), a significant increase of HSP70 gene expression was accompanied by evident epidermal morphological alterations. Partially, the morphological changes induced by HWE application can also be due to an occlusive effect exerted by the cream. However, we wonder that a possible explanation for this finding is that the application of HWE on irradiated epidermis immediately after the exposure to ionizing rays can help in preserving and/or restoring the epidermal resistance to further stress. Actually, mice deficient for inducible HSP70 proteins display an increased sensitivity to radiation (Hunt et al., 2004). In our experimental conditions, HSP70 up-regulation can be involved in the early and intense skin stress response mechanisms triggered by ionizing radiation, possibly by preventing protein misfolding and denaturation (Barral et al., 2004). Therefore, HSP70 up-regulation is consistent with a higher ability to respond to damage, and high levels of HSP70 mRNA in the skin may be 
correlated to a protective condition and can thus provide a subsequent defensive response (Matsumoto et al., 1995; Calini et al., 2003).

TGF- $\beta$ is a multi-functional cytokine that controls cell growth and differentiation. In mammals, three isoforms were isolated, among which TGF- $\beta 1$ is the best characterized (Roberts et al., 1993; Martin et al., 1997). TGF- $\beta 1$ activity varies according to the cell type and to the tissue context and, in keratinocytes, it is involved in their initiation/differentiation by blocking their proliferation (Fuchs et al., 1994; Doi et al., 2003). Our data show almost unchanged TGF- $\beta 1$ gene expression in the early phases of radiation skin response in all the experimental groups and is consistent with previous data (Donetti et al., 2005) suggesting that TGF- $\beta 1$ may play a role in later phases of the radiation skin response. Sivan and coll. studied, from a histopathological point of view, the scarring epidermis of patients from 7 months to 27 years after gamma-rays exposure (Sivan et al., 2002). In this study, suprabasal activation of keratinocytes, epidermal hyperproliferation, abnormal keratinocyte terminal differentiation, and TGF- $\beta 1$ overexpression were described.

HWE administration appeared to modulate HSP70 and TGF- $\beta$ expression in irradiated skin. Among HWE components, hyaluronic acid (HA) is a major constituent of the extracellular matrix of the skin. HA displays remarkable rheological, viscoelastic and hygroscopic properties which are relevant for dermal tissue function. Topical applications of HA improved wound healing, particularly in acute radioepithelitis (Weindl et al., 2004), and its hygroscopic properties restore the normal free water balance of the derma, lost during radiotherapy. Liguori and coll. demonstrated in a randomized, double-blind, placebo-controlled study that the prophylactic use of a cream containing $0.2 \% \mathrm{HA}$ during RT postpones the early signs of skin reactions, thus improving the compliance and quality of life in patients (Liguori et al., 1997). This component can thus play a central role in the induction of a protective condition in irradiated skin in our experimental model.

Vitis vinifera extracts are known for their antibacterial, antiviral, anticarcinogenic and antiinflammatory actions. Moreover, the presence of epigallocatechin gallate in vitis vinifera extract exerts anti-degenerative effects (Cooper et al., 2005; F'guyer et al., 2003).
Telmesteine (3-ethoxy-carbonyl-4-carboxyl-1thia-3-aza-cyclopentane) is an anti-oxidant. Its association with vitis vinifera has demonstrated significant activity against the enzymes responsible for the degeneration of the derma. Glycyrrhetinic acid is a triterpene glycoside that protects from oxidantmediated toxicity and displays antiinflammatory and antiviral properties. Both glycyrrhetinic acid (Armanini et al., 2002) and bisabolol (chamomile component) (O'Hara et al., 1998) can reduce the inflammation caused by radiotherapy.

Altogether, our results suggest that a topical treatment with HWE in irradiated skin can elicit a very early skin response inducing a protective condition aimed at counteracting damage triggered by ionizing radiation in the late phases of $\mathrm{RT}$.

\section{Acknowledgements}

We are indebted to Dr. Marchesini (Istituto Nazionale dei Tumori, Milano) and to his staff for the availability of $\gamma$-irradiator.

\section{References}

Archambeau J0, Pezner R, Wasserman T. Pathophysiology of irradiated skin and breast. Int $J$ Radiat Oncol Biol Phys 1995; 31:1171-85.

Armanini D, Fiore C, Mattarello MJ, Bielenberg J, Palermo M. History of the endocrine effects of licorice. Exp Clin Endocrinol Diabetes 2002; 110:257-61.

Bäckvall H, Wassberg C, Berne B, Ponten F. Similar UV responses are seen in a skin organ culture as in human skin in vivo. Exp Dermatol 2002;11:349-56.

Barkham AM. Radiotherapy skin reactions and treatments. Prof Nurse 1993; 8:732-6.

Barral JM, Broadley SA, Schaffar G, Hartl FU. Roles of molecular chaperones in protein misfolding diseases. Semin Cell Dev Biol 2004; 15:17-29.

Bedoni M, Sforza C, Dolci C, Donetti E. Proliferation and differentiation biomarkers in normal human breast skin organotypic cultures. J Dermatol Sci 2007; 46:139-42.

Bolderston A, Llyd NS, Wong RKS, Holden L, Robb-Blenderman L, and the Supportive Care Guidelines Group of Cancer Care Ontario Program in Evidence-based Care. The prevention and management of acute skin reactions related to radiation therapy: a systematic review and practice guidelines. Support Care Cancer 2006; 14:802-17.

Calini V, Urani C, Camatini M. Overexpression of HSP70 is induced by ionizing radiation in $\mathrm{C} 3 \mathrm{H} 10 \mathrm{~T} 1 / 2$ cells and protects fron DNA damage. Toxicol in vitro 2003; 17:561-6.

Cooper R, Morre DJ, Morre DM. Medicinal benefits of green tea: Part I. Review of noncancer health benefits. J Altern Complement Med 2005; 11:521-8.

Daugaard M, Rohde M, Jaatela M. The heat shock protein 70 family: highly homologus proteins with overlapping and distinct functions. FEBS Letters 2007; 581: 3702-10.

De Conno F, Ventafridda V, Saita L. Skin problems in advanced and terminal cancer patients. J Pain Symptom Manage 1991; 6:24756.

Doi $H$, Shibata M, Kiyokane $K$, Otsuki Y. Downregulation of TGF $\beta$ isoforms and their receptors contributes to keratinocyte hyperpro- 
liferation in psoriasis vulgaris. J Dermatol Sci 2003; 33:7-16.

Donetti E, Bedoni M, Boschini E, Bertelli AAE, Sforza C, Gagliano N. Early epidermal response after a single dose of Á-rays in organotypic culture of human breast skin. Br J Dermatol 2005; 153: 881-6.

F'guyer S, Afaq F, Mukhtar H. Photochemoprevention of skin cancer by botanical agents. Photodermatol Photoimmunol Photomed 2003; 19:56-72.

Fuchs $\mathrm{E}$, Byrne $\mathrm{C}$. The epidermis: Rising to the surface. Curr Opin Genet Dev 1994; 4:725-36.

Hunt CR, Dix DJ, Sharma GG, Pandita RK, Gupta A, Funk M, et al. Genomic instability and enhanced radiosensitivity in $\mathrm{Hsp} / 70.1$ and Hsp70.3-deficient mice. Mol Cell Biol 2004; 24:899-911.

King KB, Nail LM, Kreamer K, Strohl RA, Johnson JE. Patients descriptions of the experience of receiving radiation therapy. Oncol Nurs Forum 1985; 12:55-61.

Kwon SB, Young C, Kim DS, Choi HO, Kim KH, Chung JH et al. Impaired repair ability of hsp70.1 KO mouse after UVB irradiation. J Dermatol Sci 2002;144-51.

Lavery BA: Skin care during radiotherapy: a survey of UK practice. Clin Oncol (R Coll Radiol) 1995; 7:184-7.

Liguori V, Guillemin C, Pesce GF, Mirimanoff RO, Bernier J. Doubleblind, randomized clinical study comparing hyaluronic acid cream to placebo in patients treated with radiotherapy. Radiother Oncol 1997; 42: 155-61.

Lindquist S. The heat-shock response. Annu Rew Biochem 1986; 55:1151-91.

Martin M, Vozenin MC, Gault N, Crechet F, Pfarr CM, Lefaix JL. Coactivation of AP-1 activity and TGF-betal gene expression in the stress response of normal skin cells to ionizing radiation. Oncogene 1997; 15:981-9.

Matsumoto $\mathrm{H}$, Wang $X$, Ohnishi T. Binding between wild-type $\mathrm{p} 53$ and HSP72 accumulated after UV and $\gamma$-rays irradiation. Cancer Lett 1995; 92:127-33.
Maytin EV. Heat shock proteins and molecular chaperones: implications for adaptive responses in the skin. J Invest Dermatol 1995; 104:448-55

Moll I, Houndek P, Schafer S, Nuber U, Moll R. Diversity of desmosomal proteins in regenerating epidermis: immunohistochemical study using a human skin organ culture model. Arch Dermatol Res 1999; 291:437-446.

O'Hara M, Kiefer D, Farrell K, Kemper K. A review of 12 commonly used medicinal herbs. Arch Fam Med 1998; 7:523-36.

Porock D, Kristjanson L. Skin reactions during radiotherapy for breast cancer: the use and impact of topical agents and dressings. Eur J Cancer Care 1999; 8:143-53.

Porock D. Factors influencing the severity of radiation skin and oral mucosal reactions: development of a conceptual framework. Eur $\mathrm{J}$ Cancer Care 2002; 11:33-43.

Roberts AB, Sporn MB. Physiological actions and clinical applications of transforming growth factor-beta (TGF-beta). Growth Factors 1993; 8:1-9.

Schmuth M, Wimmer MA, Hofer S, Sztankay A, Weinlich G, Linder $D M$ et al. Topical corticosteroid therapy for acute radiation dermatitis: a prospective, randomized, double-blind study. $\mathrm{Br} J$ Dermatol 2002; 146:983-91.

Sivan V, Vozenin-Brotons MC, Tricaud Y, Lefaix JL, Cosset JM, Dubray $B$ et al. Altered proliferation and differentiation of human epidermis in cases of skin fibrosis after radiotherapy. Int $\mathrm{J}$ Radiat Oncol Biol Phys 2002; 53:385-93.

Weind $G$, Schaller M, Schafer-Korting M, Korting HC. Hyaluronic acid in the treatment and prevention of skin diseases: molecular biological, pharmaceutical and clinical aspects. Skin Pharmacol Physiol 2004; 17:207-13.

Williams MS, Burk M, Loprinzi CL, Hill M, Schomberg PJ, Nearhood $\mathrm{K}$ et al. Phase III double-blind evaluation of an aloe vera gel as a prophylactic agent for radiation-induced skin toxicity. Int J Radiat Oncol Biol Phys 1996; 36:345-9. 
N. Gagliano et al. 